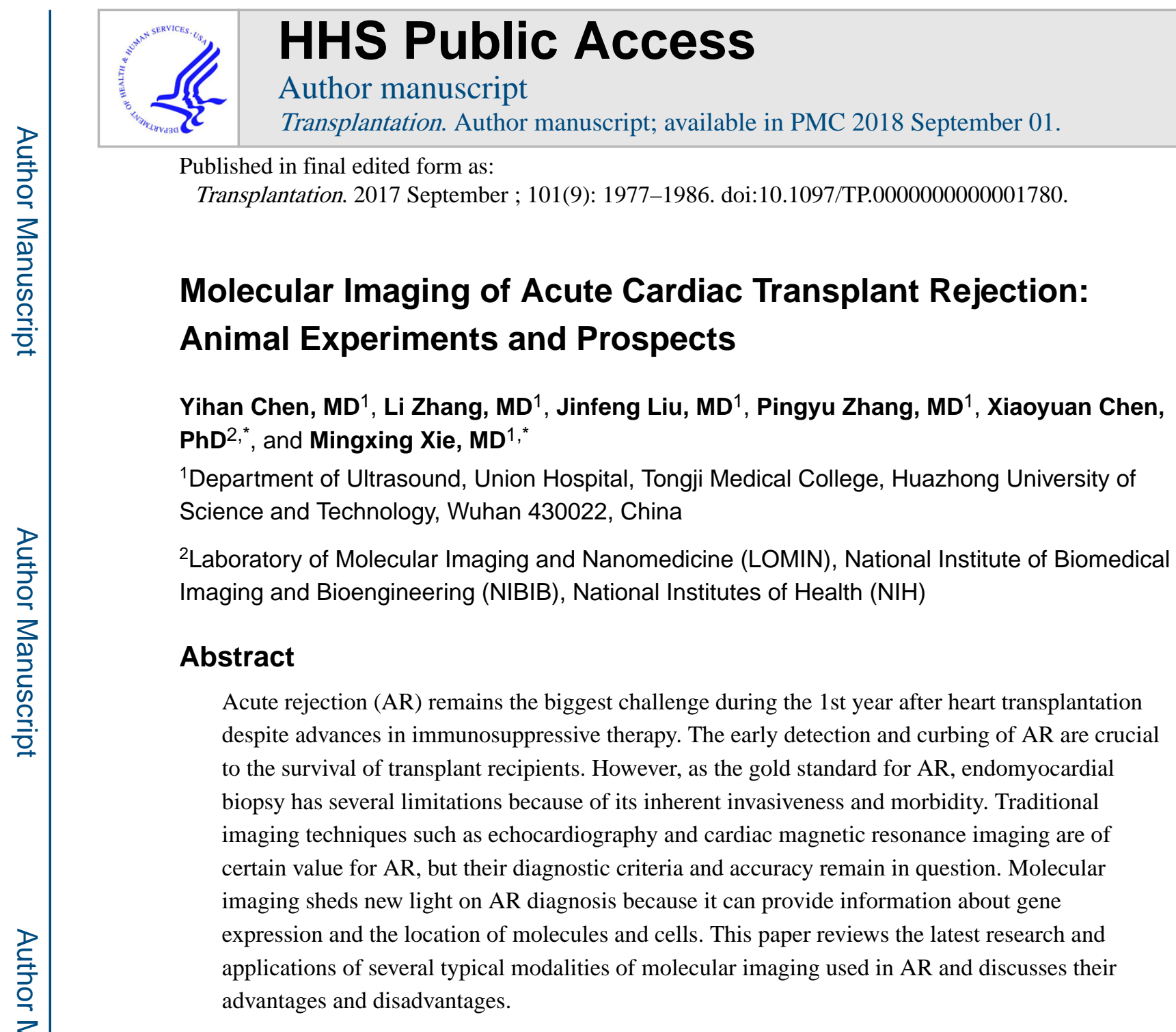

\title{
Introduction
}

Heart transplantation currently achieves great success in patients with end-stage heart failure. However, acute rejection (AR) after heart transplantation remains the biggest challenge with this life-saving treatment during the 1st year after transplantation.

Approximately $40 \%$ of patients will experience at least 1 episode of AR within this period. ${ }^{1}$ Furthermore, AR contributes to approximately $12 \%$ of the mortalities that occur between 1 and 12 months posttransplantation. ${ }^{2}$ Moreover, AR is an independent risk factor for the development of cardiac allograft vasculopathy, an irreversible stage on the path to final allograft dysfunction. Therefore, the early detection and curbing of AR is crucial to the survival of transplant recipients.

\footnotetext{
"Correspondence: Xiaoyuan Chen, PhD, Laboratory of Molecular Imaging and Nanomedicine (LOMIN), National Institute of Biomedical Imaging and Bioengineering (NIBIB), National Institutes of Health (NIH), Bethesda, Maryland 20892, United States. (Shawn.chen@ nih.gov). Mingxing Xie, MD, Department of Ultrasound, Union Hospital, Tongji Medical College, Huazhong University of Science and Technology, 1277 JieFang Avenue, Wuhan 430022, China. (xiemx64@126.com).

Author Contributions: Yihan Chen conceived of and wrote the manuscript. Li Zhang, Jinfeng Liu and Pingyu Zhang collected and analyzed the data. Mingxing Xie and Xiaoyuan Chen provided critical appraisal of the manuscript, expertise, review and editorial assistance.
}

Conflicts of Interest: The authors declare no conflicts of interest. 
At present, endomyocardial biopsy (EB) is the only gold standard for AR diagnosis. However, as an invasive procedure, routine EB is extremely inconvenient for the patient and is concomitant with several major, albeit rare, complications, such as carotid artery puncture, permanent heart block and cardiac tamponade. Furthermore, EB has disadvantages including radiation exposure, sample error, myocardial scarring and venous thrombosis. ${ }^{3,4}$ For this reason, the strategy of performing EB 1 year after transplantation has been questioned, and attempts to reduce its frequency have also been reported. ${ }^{5}$ Therefore, a noninvasive and facile evaluation method for the detection of rejection in cardiac transplant patients is highly desirable.

Nowadays, a noninvasive gene expression profiling (GEP) test (AlloMap, CareDx, Brisbane, California) has been already well tested and clinical accepted for surveillance after heart transplantation in selected low risk patients with respect to clinical outcomes. ${ }^{6}$ The test is performed on a blood sample, reported as a single score that mainly exclude the likelihood of cellular rejection. The guidelines of International Society of Heart and Lung Transplantation stated that GEP test can be used to rule out the presence of acute cellular rejection of grade $2 \mathrm{R}$ or greater in appropriate low-risk patients, between 6 months and 5 years after heart transplantation. ${ }^{7}$ However, The test just excels in regard to a very high negative predictive value. The positive predictive value for cellular rejection is not high. And it can't apply to other forms of heart rejections such as antibody-mediated rejection or cardiac allograft vasculopathy. Image technologies that can noninvasively provide visualized information are still needed.

Among many imaging techniques, echocardiography and cardiac magnetic resonance (CMR) imaging are considered the most promising diagnostic techniques to detect AR. Furthermore, some studies have confirmed that the diagnostic accuracy of echocardiography and CMR for assessing the rejection grade of AR is strongly correlated with EB. ${ }^{8}$ However, due to methodological heterogeneity, the use of different parameters and cut-off values have led to conflicting outcomes between studies, thus limiting the clinical application of echocardiography and CMR. More importantly, diagnosis using traditional imaging techniques depends on morphological and hemodynamic changes. By the time that these appear, AR has already entered a "relatively serious stage". To achieve better long-term results, diagnosis methods that can be used at earlier stages are urgently required. ${ }^{2}$

In recent years, molecular imaging has improved greatly and has shed new light on AR diagnosis. Molecular imaging represents the convergence of multiple image-capture techniques, basic cell/molecular biology, chemistry, medicine, pharmacology, medical physics, biomathematics, and bioinformatics into a new imaging paradigm. ${ }^{9}$ With the help of specific molecular probes known as biomarkers, we can visualize cellular function and follow molecular processes in vivo, enabling earlier and more precise diagnosis before morphological and hemodynamic damage occur. Molecular imaging is currently applied to the diagnosis of diseases such as cancer, neurological and cardiovascular disease, and AR.

Many modalities can be used for the noninvasive molecular imaging of AR, such as ultrasound, magnetic resonance imaging (MRI), nuclear imaging, including positron emission tomography (PET) and single photon emission-computed tomography (SPECT), 
and optical imaging. Each technique has its own strengths and weaknesses, and some are more adept at imaging multiple targets than others. In this article, we attempted to further assess the diagnostic value of several typical molecular imaging modalities for AR. Since most current studies are limited to animals, rarely refer to human (Table 1). Thus, this review will mainly focus on animal studies.

\section{Ultrasound Molecular Imaging}

Ultrasound molecular imaging (UMI) has emerged as a promising noninvasive imaging strategy for assessing biological processes at the molecular level and combines the advantages of ultrasonography (including high spatial and temporal resolution, lack of ionizing irradiation, relatively low cost, and widespread availability in imaging departments throughout the world) with novel molecular-targeted ultrasound contrast agents. ${ }^{10}$ Molecular ultrasound contrast agents are micro- or nano-sized gas bubbles that are stabilized by a surfactant or polymer coating (Figure 1). When high-affinity binding ligands are added onto the bubble shell, molecular ultrasound contrast agents can bind to specific receptors that are involved in various disease processes. After intravenous administration, these targeted ultrasound contrast agents accumulate at tissue sites that overexpress specific molecular markers, thereby enhancing the ultrasound imaging signal.

Weller et al, were the 1st to apply UMI to the detection of acute cardiac transplant rejection. ${ }^{11}$ Using anti-intercellular adhesion molecule-1 antibody-conjugated microbubbles $\left(\mathrm{MB}_{\mathrm{ICAM}}\right)$, the authors assessed the inflammatory status of the endothelium, which plays a pivotal role in the pathogenesis of heart transplant rejection. After intravenous injection, $\mathrm{MB}_{\text {ICAM }}$ was selectively bound to rejecting versus nonrejecting myocardium, thus enabling the detection of acute rejection. The authors also found that the myocardial video intensity obtained using $\mathrm{MB}_{\text {ICAM }}$ was significantly higher in a rejecting group (Brown Norway to Lewis strain) than in control groups (Lewis to Lewis or Brown Norway to Brown Norway). Conversely, control myocardium showed little difference between $\mathrm{MB}_{\mathrm{ICAM}}$ and control microbubble (conjugated with isotype control antibody) adhesion. ICAM-1-targeted ultrasound signal was strongly correlated with ICAM-1 expression and histological rejection status. However, this study was limited to the extremes of normal and fulminant disease. It remains unknown whether intercellular adhesion molecule-1 expression or microbubble attachment to the molecules parallels rejection severity.

To further study this issue, Isao Kondo et al, applied leukocyte-targeted microbubbles for the quantitative assessment of acute cardiac rejection of varying severity. ${ }^{12}$ The pattern of signal intensity variation among different severities of AR agreed well with macrophage cell counts, which were correlated with CD3-positive cell (T lymphocyte) counts. BR1, an aqueous suspension of stabilized sulfur hexafluoride bubbles encased by lipid shells, were used as leukocyte-targeted microbubbles in their study. In a previous study, lipid microbubble agents were retained within the microcirculation of inflamed tissue due to their attachment to and phagocytosis by activated leukocytes, including macrophages.

Microbubbles that interact with leukocytes remain acoustically active and enable the ultrasound imaging of leukocytes that are recruited by reperfusion injury in the kidney and heart. ${ }^{13,14}$ 
Both of these previous studies focused on inflammatory markers in blood vessels that are also involved in other pathologic conditions, such as ischemia or reperfusion injury, which inevitably occur during heart transplantation. Therefore, the specificity and accuracy of UMI-targeted inflammatory molecular or macrophages for the diagnosis of AR remains unclear.

$\mathrm{T}$ cells play a central role in graft rejection, and the degree of $\mathrm{T}$ cell infiltration is the primary grading standard of AR in clinical heart transplantation. Thus, the infiltration of UMI-targeted T cells in rejected myocardium may improve the sensitivity and accuracy of AR diagnosis. However, activated T cells are mainly distributed in the myocardial interstitium outside blood vessels during AR. Widely used ultrasound contrast agents include micro-sized particles (1 8 $\mu \mathrm{m}$ in diameter), similar to the size of erythrocytes. ${ }^{15}$ Therefore, microbubbles are used only in blood pool imaging modalities because they cannot penetrate blood vessels. To image T cells outside blood vessels, small-sized bubbles are urgently required. The development of nanotechnology in molecular imaging provides a possible solution. Nanoscale bubbles (nanobubbles) that are less than $700 \mathrm{~nm}$ in diameter can penetrate the vessel wall, which makes extravascular tissue imaging possible. ${ }^{16}$ Nanobubbles are composed of various polymer or phospholipid shells and gas, liquid or solid cores. Due to the enhanced permeation and retention effects that such bubbles exert at the sites of tumor vascular leaks with pore sizes of up to $780 \mathrm{~nm}$, nanobubbles have been extensively applied in extravascular ultrasonic imaging in various diseases. ${ }^{17,18}$ Wei Wu et al, used phospholipid shell and gas core nanobubbles that were loaded with anti-CD25 antibodies that targeted activated T cells for the ultrasound molecular imaging of AR. ${ }^{19}$ Compared with control nanobubbles, CD25-targeted nanobubbles showed a delayed enhancement of myocardium and a 2nd peak in the time-intensity curve. The intensity and time of the 2nd peak were both positively correlated with transplant time and the AR pathologic grade.

\section{Molecular and Cellular MRI}

Molecular and cellular MRI is a noninvasive technique for the visualization of molecular and cellular processes in vivo using molecular probes (Figure 2). Due to its high spatial resolution, excellent soft tissue contrast and ability to detect and quantify molecular probes, molecular MRI has been used to image cardiovascular morphology, physiology, and molecular events, including AR.

Iron oxide-based contrast agents (table 1), such as superparamagnetic iron oxide (SPIO, with particle sizes ranging from $>30$ to $1000 \mathrm{~nm}$ in diameter), ultrasmall superparamagnetic iron oxide (USPIO, with particle sizes ranging between 10 and $30 \mathrm{~nm}$ in diameter) nanoparticles, and microsized iron oxide particles (MPIO, $<10 \mu \mathrm{m}$ ), have been used to label and track cells in vivo using T2*-weighted MRI (also termed cellular MRI). Most cellular MRI studies of AR rely on the superior relaxivity of SPIO or USPIO nanoparticles for image contrast. ${ }^{20,21}$ It is thought that SPIO and USPIO particles are taken up by immune cells, mainly by macrophages. Using these agents, macrophages can be labeled in vivo by direct intravenous injection, and the degree of image contrast due to iron-oxide-labeled cells correlates well with rejection grade determined by histology. ${ }^{22}$ Because of the small particle size, cells must 
ingest thousands of SPIO or USPIO particles to create a local magnetic field gradient that is detectable by T2*-weighted MRI, especially at the level of a single cell. Each micrometersized MPIO can contain picogram quantities of iron and exerts a much greater magnetic susceptibility effect than a smaller particle. Therefore, the loading of 1 or a few MPIO particles into a cell is sufficient for MRI detection. ${ }^{23,24}$ Electron micrograph studies have shown that MPIOs are concentrated by macrophages within membrane-bound vesicles. The macrophage thus becomes the basic unit of contrast. Individual cells can be easily detected by MRI due to the superparamagnetic iron center, which can propagate a magnetic field gradient over an area as much as 50 times that of the particle radius. ${ }^{25}$

Furthermore, cells can not only be labeled in vivo but also ex vivo. ${ }^{26}$ The in vivo cell labeling efficiency of iron oxide by direct intravenous injection is low because of particle dilution and accessibility. However, cells can be isolated from a host and labeled with a suitable MRI contrast agent ex vivo in a culture medium. The labeled cells can then be infused into an animal or a human, and their migration can be monitored by MRI. In this way, more contrast agent can be endocytosed by cells, resulting in a higher labeling efficiency. This method is especially suitable for smaller particles, such as SPIO and USPIO particles. Additionally, the labeling of nonspecific cell types can be avoided using an ex vivo procedure. However, ex vivo labeling is complex, requires the isolation of cells and is challenging for in vivo cell labeling.

Iron-oxide-based cellular imaging is very sensitive; however, because this material is a negative contrast agent, the hypointense contrast produced can be confused by intrinsic contrast, image artifacts, or pathologies that also result in negative contrast. Therefore, in some cases, it is difficult to unambiguously assign contrast to labeled cells. Moreover, it is sometimes difficult in practice to robustly quantify the hypointense contrast and regions of signal dropout due to the presence of labeled cells. Due to these limitations, it would be useful to have a cellular imaging agent that provides positive image contrast or positive signal. ${ }^{27}$

Gadolinium-based MR probes generate positive contrast in T1 MRI images, which can be detected more easily than the negative contrast generated by iron oxide particles. To conduct molecular or cellular imaging, these new Gd-based MR probes are designed to conjugate a specific vector (eg, small molecules, peptides, or antibodies) with a Gd-chelate. After binding of the probe to its target, a more intense $\mathrm{T} 1$ effect can be induced. ${ }^{28}$ These probes involve Gd-containing liposomes, ${ }^{29}$ lipoproteins, ${ }^{30}$ and micelles. ${ }^{31}$ However, molecular or cellular MRI for AR that rely on gadolinium-based probes has rarely been reported.

Several techniques have been developed to generate positive contrast with iron-oxide cell labeling, such as inversion recovery with ON-resonance water suppression ${ }^{32}$ and phasesensitive techniques that enhance the detectability of iron-oxide labeled cells. ${ }^{33,34}$ However, these techniques may not achieve pervasive application due to the necessity for magnetic preparation pulses and due to difficulties at tissue interfaces or with certain pathologies that involve inherent magnetic susceptibility and image phase differences. 
Fluorine agents comprise a class of agents used for cellular MRI that have gained attention in recent years. In contrast to cellular imaging with metal-ion agents, fluorine agents are directly detected. Hitchens et al, used a commercially available formulation of perfluoro-15crown-5-ether (PCE) to label and detect macrophage accumulation in rat models of cardiac and renal rejection by ${ }^{19} \mathrm{~F}$ MRI. ${ }^{27}$ Almost no background signal is present that would need to be distinguished from the labeled cells because very low levels of fluorine are typically found in mammals (mainly as fluoride). This provides the advantage that the signal is highly correlated with labeled cells and is unlikely to be confused with other pathologies or image artifacts. While detecting macrophage accumulation due to rejection, the results of ${ }^{19} \mathrm{~F}$ MRI are generally consistent with iron-oxide cell labeling and $\mathrm{T} 2 *$-weighted contrast imaging, ${ }^{23,35}$ and these 2 techniques can offer complementary information in many respects. Iron-oxide agents offer high sensitivity at high resolution, and ${ }^{19} \mathrm{~F}$ offers unambiguous detection and a more easily quantified signal.

Most strategies used so far have mainly focused on macrophages rather than $\mathrm{T}$ cells. For example, as a prevalent agent used in cellular MRI, SPIO/USPIO particles are taken up mainly by macrophages and are not taken up to the same extent by T cells. ${ }^{36}$ In theory, cellular MRI using iron-oxide agents reflects mainly macrophage-related changes in graft rejection. Thus, ways must be found to enhance the incorporation of these MRI contrast agents into $\mathrm{T}$ cells. The conjugation of specific targeting vectors (eg, small molecules, peptides, or antibodies) with MRI contrast agents may be a favorable choice. CD3 antigen is a universal marker for T cells. Guo et al, developed a MRI-visible and CD3 antibody(scAbCD3)-targeted multifunctional polymeric nanocarrier containing SPIO for the detection of $\mathrm{T}$ cells in AR and gene delivery (Figure 3). ${ }^{37}$ Lewin $\mathrm{M}$ and Dodd $\mathrm{CH}$ prepared a new composite particle by conjugating a peptide from the transactivator protein (Tat) of HIV-1 to cross-linked aminated dextran-coated iron oxide particles. ${ }^{38,39}$ The Tat peptide has been shown to promote the translocation of various "cargos" through the plasma membrane of a variety of cells, including T cells. ${ }^{40}$ The receptor-mediated endocytosis pathway is another approach that can be used to increase the incorporation of MR contrast agents into cells. Ahrens et al, conjugated an anti-CD11c monoclonal antibody to commercially available SPIO particles and then incubated the resulting particle conjugates with bone marrow-derived dendritic cells (DCs) or with a fetal skin-derived DC line in culture media. ${ }^{41}$ These cells exhibited an approximately 50-fold increase in uptake relative to DCs that were incubated with SPIO particles without the monoclonal antibody; importantly, this labeling procedure did not affect the immunological function of the cell. The authors further reported that when these labeled DCs were injected into murine quadriceps and then monitored in vivo for several days using MRI at $11.7 \mathrm{~T}$, the SPIO-labeled DCs remained within the muscle cells for longer than $24 \mathrm{hrs}$. The receptor-mediated endocytosis pathway may provide a good way to label $\mathrm{T}$ cells for use in cellular MRI.

\section{Nuclear Imaging}

Nuclear medicine is the main application of molecular and cellular imaging in current clinical use. Positron emission tomography (PET) and single photon emission-computed tomography (SPECT) are the main molecular imaging modalities. The strengths of nuclear 
imaging are its high sensitivity and the availability of a wide variety of targeted, radioactively labelled tracers in experimental and clinical applications. ${ }^{42}$

Heart transplant rejection is characterized pathologically by myocyte necrosis and interstitial mononuclear cell infiltration-associated apoptosis. Either of these components can be targeted for the noninvasive detection of transplant rejection (Figure 4). A body of research from the 1980s and 1990s explored the use of radionuclide scintigraphy for the noninvasive detection of cardiac allograft rejection. Eisen $\mathrm{HJ}$ et al, noninvasively detected the rejection of transplanted hearts using indium-111( ${ }^{111}$ In)-labeled lymphocytes in dogs in $1987 .{ }^{43}$ In the same year, Frist W et al, further confirmed that imaging with ${ }^{111}$ In-labeled antimyosin antibody Fab fragments can be valuable for the noninvasive identification of rejection in cardiac transplant recipients. ${ }^{44}$ Approximately 10 years later, the feasibility of radionuclide scintigraphy using ${ }^{111}$ In-labeled lymphocytes for diagnosing AR in human subjects was confirmed by Rubin PJ et al. ${ }^{45}$ Additionally, ${ }^{99} \mathrm{~m}_{\text {technetium-labeled annexin-V (a marker of }}$ apoptosis), ${ }^{46,47}$ oligonucleotides against interleukin- 2 mRNA, ${ }^{48}$ and anti-sialoadhesin mAb (a marker of inflammatory macrophages) have been used for the detection of AR (Figure 5). ${ }^{49}$

Currently, the leading molecular imaging modality is PET. The spatial resolution of PET is higher than that of SPECT (4-5 vs. 10-16 mm with clinical scanners). Furthermore, attenuation (the quantifiable reduction in the number of events present at the face of the detector due to absorption or scattering through tissues) of the emitted radiation in PET can be precisely corrected because the total length through the body determines the attenuation factor along a coincidence line. Therefore, PET is a more robust technique than SPECT for imaging most molecular events.

Compared to other conventional modalities such as MRI, both PET and SPECT offer limited resolution, although PET is better. Further investigation is needed regarding the use of more powerful gamma emitters, such as ${ }^{18} \mathrm{~F}$, which yield higher spatial resolution. The glucose analogue 2-[ $\left.{ }^{18} \mathrm{~F}\right]$ fluoro-2-deoxy-D-glucose $\left(\left[{ }^{18} \mathrm{~F}\right] \mathrm{FDG}\right)$ is a widely used tracer that is taken up by all glucose-metabolizing cells, including leukocytes, myocardial cells, and neuronal cells. There is a hypothesis that rejection shifts myocardial metabolism toward glucose in general or increases the uptake of $\left[{ }^{18} \mathrm{~F}\right] \mathrm{FDG}$ relative to glucose. ${ }^{50}$ Thus, in theory, $\left[{ }^{18} \mathrm{~F}\right] \mathrm{FDG}$ can be used to quantify glucose uptake and glycolytic activity in transplanted hearts. ${ }^{51}$ Daly $\mathrm{KP}$ et al, used ${ }^{18} \mathrm{~F}$-labeled fluorodeoxyglucose ([ $\left.\left.{ }^{18} \mathrm{~F}\right] \mathrm{FDG}\right)$ to monitor the evolution of cardiac allograft rejection in a murine model. ${ }^{52}$ Combined with the use of $\left[{ }^{13} \mathrm{~N}\right] \mathrm{NH} 3 \mathrm{PET}$ imaging, which reflects myocardial blood flow, this method can be used as a noninvasive, quantitative technique for the serial monitoring of allograft rejection and has potential application in human transplant recipients. However, due to the immune responses that coincide with ischemia-reperfusion injury and the working condition of the heart, the basal myocardial uptake of $\left[{ }^{18} \mathrm{~F}\right] \mathrm{FDG}$ is variable, and this may ultimately affect the ability of this technique to detect rejection. ${ }^{53,54}$

Another means of enhancing the resolution of these technologies is to use multiple modalities. The simplest manifestation of this is the coregistration of images obtained lowresolution techniques (for example, PET) with high-resolution anatomical images (such as 
those obtained, for example, using CT or MRI) obtained in the same session. Thus, functional imaging by PET or SPECT, which depicts the spatial distribution of metabolic or biochemical activity in the body, can be more precisely aligned or correlated with anatomical images obtained using CT or MRI. Ueno T et al, detected macrophage infiltration in cardiac mouse allografts using copper-64-derivatized dextran nanoparticle PET/CT imaging. The authors also demonstrated that this method can be used to monitor therapeutic immunomodulation in mice with total allo-mismatched hearts. ${ }^{55}$

Although much evidence has shown the reliability of molecular imaging for AR detection by PET or SPECT, these techniques are considered to decrease but not eliminate the need for protocol biopsies. It is likely that a negative PET or SPECT scan could allow for a decreased biopsy frequency, whereas a positive PET or SPECT scan would trigger a biopsy.

Furthermore, exposure to ionizing radiation is a limitation that might play an important role in the screening of asymptomatic individuals or in repeated, follow-up imaging.

Furthermore, it should be noted that the animal model most commonly used in AR research is heterotopic transplantation into the abdominal cavity; this model results in a beating but nonworking heart that contacts the peritoneum. Therefore, translating results from this model to heart transplantation requires caution.

\section{Optical Molecular Imaging}

Currently, optical molecular imaging (OMI) has become 1 of the most widely used molecular imaging techniques. With the use of specific molecular markers (such as luciferase and fluorescent proteins), OMI has been applied to imaging fundamental biological processes in vivo, such as in pathogenesis research, gene expression regulation and activity detection, cytological detection, pharmaceutical effect evaluation and efficacy evaluation (Figure 6). ${ }^{56}$

OMI includes a variety of imaging modalities, such as bioluminescent imaging (BLI), fluorescence imaging (FI), optical coherence tomography (OCT), bioluminescent tomography (BLT), fluorescence molecular tomography (FMT), laser speckle imaging, polarization imaging, fluorescence reflectance imaging (FRI), diffuse optical tomography (DOT), fluorescence resonance imaging, and NIRF. ${ }^{57} \mathrm{BLI}$ and FI are the most commonly used OMI techniques.

The 1st approach to detecting allograft rejection in mice using an optical technique involved bioluminescence imaging. Tanaka M and colleagues used in vivo BLI to visualize changes in cardiac allograft viability and donor-derived passenger CD5+ cells that occurred in response to cardiac allografting during acute rejection. ${ }^{58}$ The authors found that 4 days after transplantation, the intensity of light emitted from cardiac allografts declined in a manner that was correlated with graft beating scores. Furthermore, the light signal from CD5+ passenger leukocytes peaked at 6 hours and as decreased at 12 hours in allograft recipients, whereas the signal remained high until 10 days after transplantation in syngeneic recipients. This research showed that BLI is a useful modality for the quantitative assessment of in vivo cardiac graft viability and the tracking of passenger leukocytes in vivo during acute rejection. Unlike the use of graft beating score, which assesses only whether the cardiac 
allograft is beating or not, BLI can assess cardiac graft viability and is more suitable for determining cardiac graft survival.

$\mathrm{NF}-\kappa \mathrm{B}$ is a potent proinflammatory signal transduction molecule that plays a key role in regulating the expression of multiple chemokine and cytokine genes that are involved in innate and adaptive immune responses. ${ }^{59}$ Lianli Ma and coworkers used BLI to detect NF$\kappa \mathrm{B}$ activation in a cardiac transplantation mouse model and tissue ischemia-reperfusion injury. ${ }^{60}$ The results that they obtained showed that luciferase activity was significantly increased in the cardiac allografts and was reduced after CD154 mAb therapy. This study indicated that BLI may be a viable approach to noninvasively and quantitatively visualize $\mathrm{NF}-\kappa \mathrm{B}$ activation within passenger leukocytes and recipient lymphocytes in a cardiac transplantation mouse model.

FI is another optical technique that has been used in acute rejection. Thomas Christen et al, injected allograft recipient mice with a quenched fluorescent substrate reporter for cysteinyl proteases, which are expressed abundantly by leukocytes that accumulate in allografts. ${ }^{61} \mathrm{In}$ vivo fluorescence molecular tomography imaging of the macrophage response is correlated closely with gradually increasing allograft rejection and attenuated rejection in recipients with a genetically impaired immune response that results from a deficiency in recombinase-1 (RAG- $1^{-/-}$), indicating that fluorescence molecular imaging can noninvasively detect protease activity in cardiac allograft rejection and has translational potential.

The advantages of OMI include high specificity and sensitivity, use of nonionizing radiation, and the ability to label many important genes and proteins. However, the overall low spatial resolution and limited penetration depth of optical imaging signals has limited its clinical application. New approaches, such as fluorescence molecular tomography, which allows the volumetric reconstruction of the source of fluorescent light, can improve spatial resolution to $1 \mathrm{~mm}$ at the surface to $3 \mathrm{~mm}$ in the center of small animals. ${ }^{62}$ However, this technique is insufficient for human cardiac imaging. Near infrared imaging may be an applicable choice because the wavelength used ranges from 650 to $900 \mathrm{~nm}$, which can in theory penetrate 7$14 \mathrm{~cm}$ of tissue. ${ }^{63}$ However, the spatial resolution remains to be improved. Furthermore, similar to nuclide imaging techniques such as SPECT and PET, it is difficult to obtain structural images of biological tissue.

\section{Conclusions}

Molecular and cellular imaging has shown great potential for transplantation. The common modalities of molecular and cellular imaging, including magnetic, optical, nuclear, and ultrasound methods, applied to different cellular and molecular targets all allow the detection of allograft rejection. However, each of these methods has strengths and weaknesses. The sensitivities of nuclear imaging and OMI are high, but the spatial resolution is low. MRI and ultrasound methods have high spatial resolution and excellent soft tissue contrast, but the inherent sensitivity is limited. It remains unclear which approach represents the best technique for diagnosing AR. 
Until now, some of these techniques have been applied in humans. For example, nuclear imaging techniques using multiple tracers have strong advantages in terms of AR imaging in clinical practice. Additionally, iron-oxide nanoparticles have achieved approval for human use in some countries, and cardiac MRI combined with iron-oxide contrast agents seems readily transferable to the clinical diagnosis of AR. The 2015 European Association of Cardiovascular Imaging/Cardiovascular Imaging Department of the Brazilian Society of Cardiology recommendations for the use of cardiac imaging to assess and follow patients after heart transplantation stated that "A range of molecular components of acute graft rejection has been targeted with radionuclide scintigraphy". ${ }^{64}$ However, this technique is only recommended in clinical practice for patients who are unsuitable for stress echocardiography due to conflicting results in published studies and a lack of availability of large multicenter trial results.

In the future, multiple imaging modalities that combine the advantages of different techniques may be 1 of development trends. And the development of a multifunctional contrast agent fit for several imaging modalities may be a key problem. Thus, there is a need for chemists to develop contrast agents, for physicists to refine the imaging process, and for mathematicians to improve data analysis and handling. What's more, large clinical trials with multiple centers documenting the diagnostic and prognostic value of these techniques are imperatively needed.

\section{Acknowledgments}

The work described here was supported by National Natural Science Foundation of China (Grant Nos. 81530056, $81471678,81271582,81671705)$ and HUST Interdisciplinary Innovation Team (0118530300).

\section{List of abbreviations}

$\begin{array}{ll}\text { AR } & \text { acute rejection } \\ \text { BLI } & \text { bioluminescent imaging } \\ \text { BLT } & \text { bioluminescent tomography } \\ \text { CMR } & \text { cardiac magnetic resonance } \\ \text { DCs } & \text { dendritic cells } \\ \text { DOT } & \text { diffuse optical tomography } \\ \text { EB } & \text { endomyocardial biopsy } \\ \text { FI } & \text { fluorescence imaging } \\ \text { mAb } & \text { monoclonal antibody } \\ \text { MB } & \text { microbubbles } \\ \text { MPIO } & \text { microsized iron oxide particles } \\ \text { OCT } & \text { optical coherence tomography }\end{array}$

Transplantation. Author manuscript; available in PMC 2018 September 01. 


$\begin{array}{ll}\text { OMI } & \text { optical molecular imaging } \\ \text { SER-4 } & \text { sialoadhesin } \mathrm{mAb} \\ \text { SPIO } & \text { superparamagnetic iron oxide } \\ \text { Tat } & \text { transactivator protein } \\ \text { UMI } & \text { Ultrasound molecular imaging } \\ \text { USPIO } & \text { ultrasmall superparamagnetic iron oxide }\end{array}$

\section{References}

1. Patel JK, Kobashigawa JA. Should we be doing routine biopsy after heart transplantation in a new era of anti-rejection? Curr Opin Cardiol. 2006; 21(2):127-131. [PubMed: 16470149]

2. Stehlik J, Edwards LB, Kucheryavaya AY, et al. The Registry of the International Society for Heart and Lung Transplantation: twenty-seventh official adult heart transplant report--2010. J Heart Lung Transplant. 2010; 29(10):1089-1103. [PubMed: 20870164]

3. Spiegelhalter DJ, Stovin PG. An analysis of repeated biopsies following cardiac transplantation. Stat Med. 1983; 2(1):33-40. [PubMed: 6359316]

4. Baraldi-Junkins C, Levin HR, Kasper EK, Rayburn BK, Herskowitz A, Baughman KL. Complications of endomyocardial biopsy in heart transplant patients. J Heart Lung Transplant. 1993; 12(1 Pt 1):63-67. [PubMed: 8443204]

5. From AM, Maleszewski JJ, Rihal CS. Current status of endomyocardial biopsy. Mayo Clin Proc. 2011; 86(11):1095-1102. [PubMed: 22033254]

6. Crespo-Leiro MG, Stypmann J, Schulz U, et al. Clinical usefulness of gene-expression profile to rule out acute rejection after heart transplantation: CARGO II. Eur Heart J. 2016; 37(33):25912601. [PubMed: 26746629]

7. Costanzo MR, Dipchand A, Starling R, et al. The International Society of Heart and Lung Transplantation Guidelines for the care of heart transplant recipients. J Heart Lung Transplant. 2010; 29(8):914-956. [PubMed: 20643330]

8. Dodd DA, Brady LD, Carden KA, Frist WH, Boucek MM, Boucek RJ Jr. Pattern of echocardiographic abnormalities with acute cardiac allograft rejection in adults: correlation with endomyocardial biopsy. J Heart Lung Transplant. 1993; 12(6 Pt 1):1009-1017. [PubMed: 8312301]

9. Massoud TF, Gambhir SS. Molecular imaging in living subjects: seeing fundamental biological processes in a new light. Genes Dev. 2003; 17(5):545-580. [PubMed: 12629038]

10. Deshpande N, Needles A, Willmann JK. Molecular ultrasound imaging: current status and future directions. Clin Radiol. 2010; 65(7):567-581. [PubMed: 20541656]

11. Weller GE, Lu E, Csikari MM, et al. Ultrasound imaging of acute cardiac transplant rejection with microbubbles targeted to intercellular adhesion molecule-1. Circulation. 2003; 108(2):218-224. [PubMed: 12835214]

12. Kondo I, Ohmori K, Oshita A, et al. Leukocyte-targeted myocardial contrast echocardiography can assess the degree of acute allograft rejection in a rat cardiac transplantation model. Circulation. 2004; 109(8):1056-1061. [PubMed: 14967733]

13. Lindner JR, Song J, Xu F, et al. Noninvasive ultrasound imaging of inflammation using microbubbles targeted to activated leukocytes. Circulation. 2000; 102(22):2745-2750. [PubMed: 11094042]

14. Christiansen JP, Leong-Poi H, Klibanov AL, Kaul S, Lindner JR. Noninvasive imaging of myocardial reperfusion injury using leukocyte-targeted contrast echocardiography. Circulation. 2002; 105(15):1764-1767. [PubMed: 11956115]

15. Ferrara KW, Borden MA, Zhang H. Lipid-shelled vehicles: engineering for ultrasound molecular imaging and drug delivery. Acc Chem Res. 2009; 42(7):881-892. [PubMed: 19552457] 
16. Yin $\mathrm{T}$, Wang $\mathrm{P}$, Zheng R, et al. Nanobubbles for enhanced ultrasound imaging of tumors. Int $\mathrm{J}$ Nanomedicine. 2012; 7:895-904. [PubMed: 22393289]

17. Hobbs SK, Monsky WL, Yuan F, et al. Regulation of transport pathways in tumor vessels: role of tumor type and microenvironment. Proc Natl Sci U S A. 1998; 95(8):4607-4612.

18. Marxer EE, Brussler J, Becker A, et al. Development and characterization of new nanoscaled ultrasound active lipid dispersions as contrast agents. Eur J Pharm Biopharm. 2011; 77(3):430437. [PubMed: 21147221]

19. Wu W, Zhang Z, Zhuo L, et al. Ultrasound molecular imaging of acute cellular cardiac allograft rejection in rat with T-cell-specific nanobubbles. Transplantation. 2013; 96(6):543-549. [PubMed: 23921443]

20. Bulte JW, Duncan ID, Frank JA. In vivo magnetic resonance tracking of magnetically labeled cells after transplantation. J Cereb Blood Flow Metab. 2002; 22(8):899-907. [PubMed: 12172375]

21. Oude Engberink RD, van der Pol SM, Dopp EA, de Vries HE, Blezer EL. Comparison of SPIO and USPIO for in vitro labeling of human monocytes: MR detection and cell function. Radiology. 2007; 243(2):467-474. [PubMed: 17456871]

22. Wu YL, Ye Q, Eytan DF, et al. Magnetic resonance imaging investigation of macrophages in acute cardiac allograft rejection after heart transplantation. Circ Cardiovasc Imaging. 2013; 6(6):965973. [PubMed: 24097421]

23. Wu YL, Ye Q, Foley LM, et al. In situ labeling of immune cells with iron oxide particles: an approach to detect organ rejection by cellular MRI. Proc Natl Acad Sci U S A. 2006; 103(6):18521857. [PubMed: 16443687]

24. Ye Q, Wu YL, Foley LM, et al. Longitudinal tracking of recipient macrophages in a rat chronic cardiac allograft rejection model with noninvasive magnetic resonance imaging using micrometersized paramagnetic iron oxide particles. Circulation. 2008; 118(2):149-156. [PubMed: 18591438]

25. Williams JB, Ye Q, Hitchens TK, Kaufman CL, Ho C. MRI detection of macrophages labeled using micrometer-sized iron oxide particles. J Magn Reson Imaging. 2007; 25(6):1210-1218. [PubMed: 17520727]

26. Ho C, Hitchens TK. A non-invasive approach to detecting organ rejection by MRI: monitoring the accumulation of immune cells at the transplanted organ. Curr Pharm Biotechnol. 2004; 5(6):551566. [PubMed: 15579044]

27. Hitchens TK, Ye Q, Eytan DF, Janjic JM, Ahrens ET, Ho C. 19F MRI detection of acute allograft rejection with in vivo perfluorocarbon labeling of immune cells. Magn Reson Med. 2011; 65(4): 1144-1153. [PubMed: 21305593]

28. Caravan P. Strategies for increasing the sensitivity of gadolinium based MRI contrast agents. Chem Soc Rev. 2006; 35(6):512-523. [PubMed: 16729145]

29. Maiseyeu A, Mihai G, Kampfrath T, et al. Gadolinium-containing phosphatidylserine liposomes for molecular imaging of atherosclerosis. J Lipid Res. 2009; 50(11):2157-2163. [PubMed: 19017616]

30. Caruthers SD, Cyrus T, Winter PM, Wickline SA, Lanza GM. Anti-angiogenic perfluorocarbon nanoparticles for diagnosis and treatment of atherosclerosis. Wiley Interdiscip Rev Nanomed Nanobiotechnol. 2009; 1(3):311-323. [PubMed: 20049799]

31. Amirbekian V, Lipinski MJ, Briley-Saebo KC, et al. Detecting and assessing macrophages in vivo to evaluate atherosclerosis noninvasively using molecular MRI. Proc Natl Acad Sci U S A. 2007; 104(3):961-966. [PubMed: 17215360]

32. Stuber M, Gilson WD, Schar M, et al. Positive contrast visualization of iron oxide-labeled stem cells using inversion-recovery with ON-resonant water suppression (IRON). Magn Reson Med. 2007; 58(5):1072-1077. [PubMed: 17969120]

33. de Crespigny AJ, Roberts TP, Kucharcyzk J, Moseley ME. Improved sensitivity to magnetic susceptibility contrast. Magn Reson Med. 1993; 30(1):135-137. [PubMed: 8371668]

34. Liu S, Buch S, Chen Y, et al. Susceptibility-weighted imaging: current status and future directions. NMR Biomed. 2017; 30(4)

35. Wu YL, Ye Q, Sato K, Foley LM, Hitchens TK, Ho C. Noninvasive evaluation of cardiac allograft rejection by cellular and functional cardiac magnetic resonance. JACC Cardiovasc Imaging. 2009; 2(6):731-741. [PubMed: 19520344] 
36. Kanno S, Wu YJ, Lee PC, et al. Macrophage accumulation associated with rat cardiac allograft rejection detected by magnetic resonance imaging with ultrasmall superparamagnetic iron oxide particles. Circulation. 2001; 104(8):934-938. [PubMed: 11514382]

37. Guo Y, Chen W, Wang W, et al. Simultaneous diagnosis and gene therapy of immuno-rejection in rat allogeneic heart transplantation model using a T-cell-targeted theranostic nanosystem. ACS Nano. 2012; 6(12):10646-10657. [PubMed: 23189971]

38. Lewin M, Carlesso N, Tung $\mathrm{CH}$, et al. Tat peptide-derivatized magnetic nanoparticles allow in vivo tracking and recovery of progenitor cells. Nat Biotechnol. 2000; 18(4):410-414. [PubMed: 10748521]

39. Dodd CH, Hsu HC, Chu WJ, et al. Normal T-cell response and in vivo magnetic resonance imaging of T cells loaded with HIV transactivator-peptide-derived superparamagnetic nanoparticles. J Immunol Methods. 2001; 256(1-2):89-105. [PubMed: 11516758]

40. Vocero-Akbani A, Lissy NA, Dowdy SF. Transduction of full-length Tat fusion proteins directly into mammalian cells: analysis of T cell receptor activation-induced cell death. Methods Enzymol. 2000; 322:508-521. [PubMed: 10914043]

41. Ahrens ET, Feili-Hariri M, Xu H, Genove G, Morel PA. Receptor-mediated endocytosis of ironoxide particles provides efficient labeling of dendritic cells for in vivo MR imaging. Magn Reson Med. 2003; 49(6):1006-1013. [PubMed: 12768577]

42. Phelps ME. PET: the merging of biology and imaging into molecular imaging. J Nucl Med. 2000; 41(4):661-681. [PubMed: 10768568]

43. Eisen HJ, Eisenberg SB, Saffitz JE, Bolman RM 3rd, Sobel BE, Bergmann SR. Noninvasive detection of rejection of transplanted hearts with indium-111-labeled lymphocytes. Circulation. 1987; 75(4):868-876. [PubMed: 3030580]

44. Frist W, Yasuda T, Segall G, et al. Noninvasive detection of human cardiac transplant rejection with indium-111 antimyosin (Fab) imaging. Circulation. 1987; 76(5 Pt 2):V81-85. [PubMed: 3311460]

45. Rubin PJ, Hartman JJ, Hasapes JP, Bakke JE, Bergmann SR. Detection of cardiac transplant rejection with 111In-labeled lymphocytes and gamma scintigraphy. Circulation. 1996; 94(9 Suppl):Ii298-303. [PubMed: 8901764]

46. Kown MH, Strauss HW, Blankenberg FG, et al. In vivo imaging of acute cardiac rejection in human patients using (99m)technetium labeled annexin V. Am J Transplant. 2001; 1(3):270-277. [PubMed: 12102261]

47. Narula J, Acio ER, Narula N, et al. Annexin-V imaging for noninvasive detection of cardiac allograft rejection. Nat Med. 2001; 7(12):1347-1352. [PubMed: 11726976]

48. Gierthmuehlen M, Sommer P, Zuhayra M, et al. Imaging of acute heart-transplant rejection using 99m-Technetium labelled oligonucleotides against interleukin-2 mRNA in rats. Eur J Cardiothorac Surg. 2010; 37(5):1111-1116. [PubMed: 20116274]

49. O’Neill AS, Terry SY, Brown K, et al. Non-invasive molecular imaging of inflammatory macrophages in allograft rejection. EJNMMI research. 2015; 5(1):69. [PubMed: 26611870]

50. Taegtmeyer H, Doenst T. F-18 FDG uptake in transplanted heart. J Am Coll Cardiol. 1998; 31(6): 1441-1442. [PubMed: 9581748]

51. de Groot M, Meeuwis AP, Kok PJ, Corstens FH, Oyen WJ. Influence of blood glucose level, age and fasting period on non-pathological FDG uptake in heart and gut. Eur J Nucl Med Mol Imaging. 2005; 32(1):98-101. [PubMed: 15605289]

52. Daly KP, Dearling JL, Seto T, et al. Use of [18F]FDG positron emission tomography to monitor the development of cardiac allograft rejection. Transplantation. 2015; 99(9):e132-139. [PubMed: 25675207]

53. Huang Y, Rabb H, Womer KL. Ischemia-reperfusion and immediate T cell responses. Cell Immunol. 2007; 248(1):4-11. [PubMed: 17942086]

54. Suga H. Global cardiac function: mechano-energetico-informatics. J Biomech. 2003; 36(5):713720. [PubMed: 12695001]

55. Ueno T, Dutta P, Keliher E, et al. Nanoparticle PET-CT detects rejection and immunomodulation in cardiac allografts. Circ Cardiovasc Imaging. 2013; 6(4):568-573. [PubMed: 23771986]

56. Du W, Wang Y, Luo Q, Liu BF. Optical molecular imaging for systems biology: from molecule to organism. Anal Bioanal Chem. 2006; 386(3):444-457. [PubMed: 16850295] 
57. Chen ZY, Wang YX, Yang F, Lin Y, Zhou QL, Liao YY. New researches and application progress of commonly used optical molecular imaging technology. BioMed Res Int. 2014; 2014:429198. [PubMed: 24696850]

58. Tanaka M, Swijnenburg RJ, Gunawan F, et al. In vivo visualization of cardiac allograft rejection and trafficking passenger leukocytes using bioluminescence imaging. Circulation. 2005; 112(9 Suppl):I105-110. [PubMed: 16159800]

59. Csizmadia V, Gao W, Hancock SA, et al. Differential NF-kappaB and IkappaB gene expression during development of cardiac allograft rejection versus CD154 monoclonal antibody-induced tolerance. Transplantation. 2001; 71(7):835-840. [PubMed: 11349713]

60. Ma L, Xiang Z, Sherrill TP, et al. Bioluminescence imaging visualizes activation of nuclear factorkappaB in mouse cardiac transplantation. Transplantation. 2008; 85(6):903-910. [PubMed: 18360274]

61. Christen T, Nahrendorf M, Wildgruber M, et al. Molecular imaging of innate immune cell function in transplant rejection. Circulation. 2009; 119(14):1925-1932. [PubMed: 19332470]

62. Guo H, Yu J, He X, Hou Y, Dong F, Zhang S. Improved sparse reconstruction for fluorescence molecular tomography with L1/2 regularization. Biomed Opt Express. 2015; 6(5):1648-1664. [PubMed: 26137370]

63. Wang K, Wang Q, Luo Q, Yang X. Fluorescence molecular tomography in the second near-infrared window. Opt Exp. 2015; 23(10):12669-12679.

64. Badano LP, Miglioranza MH, Edvardsen T, et al. European association of cardiovascular imaging/ cardiovascular imaging department of the Brazilian Society of Cardiology recommendations for the use of cardiac imaging to assess and follow patients after heart transplantation. Eur Heart J Cardiovasc Imaging. 2015; 16(9):919-948. [PubMed: 26139361]

65. Conradi L, Pahrmann C, Schmidt S, et al. Bioluminescence imaging for assessment of immune responses following implantation of engineered heart tissue (EHT). J Vis Exp. 2011; (52) pii:2605.

Transplantation. Author manuscript; available in PMC 2018 September 01. 


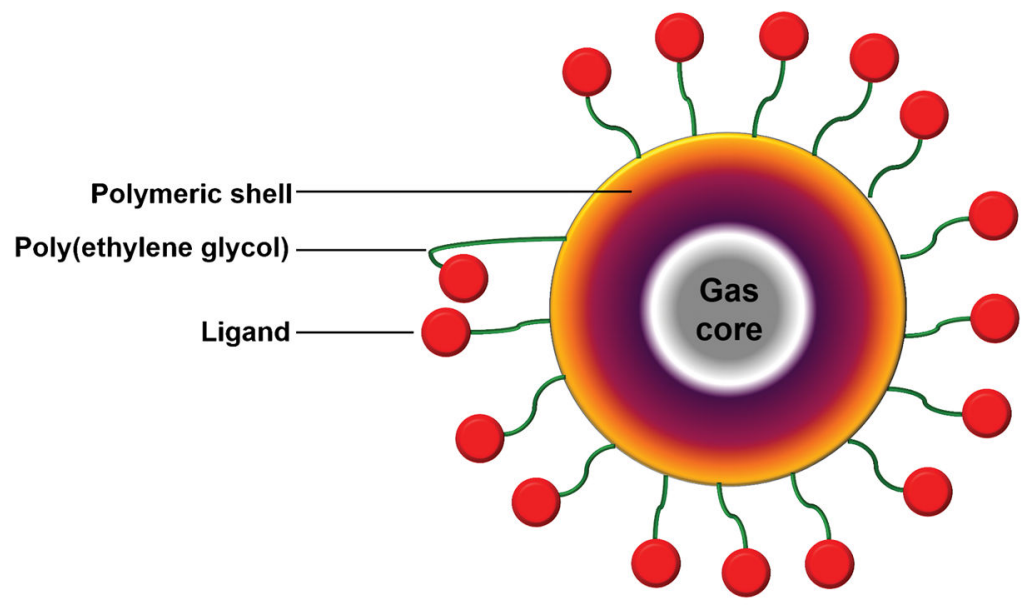

Figure 1.

Schematic diagram of a targeted microbubble. The prototype consists of a gas core encapsulated by a shell of phospholipids or other polymer conjugated with high-affinity binding ligands. 
(A)

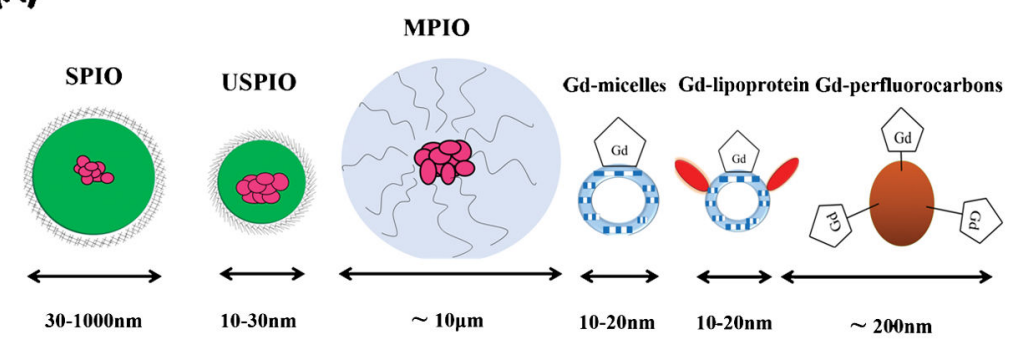

(B)

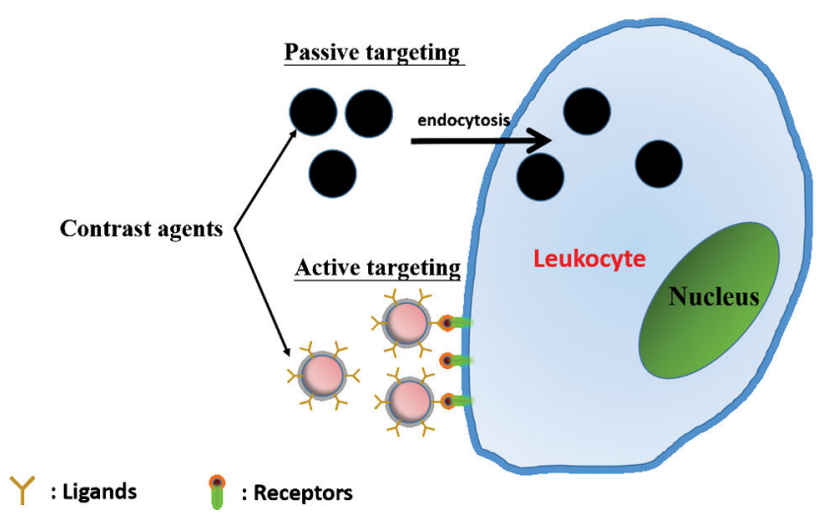

Figure 2.

(A) Molecular probes with different sizes used in molecular and cellular MRI. (B) Leukocyte is the common target of molecular and cellular MRI in AR. Passive or active targeting strategies are used. 

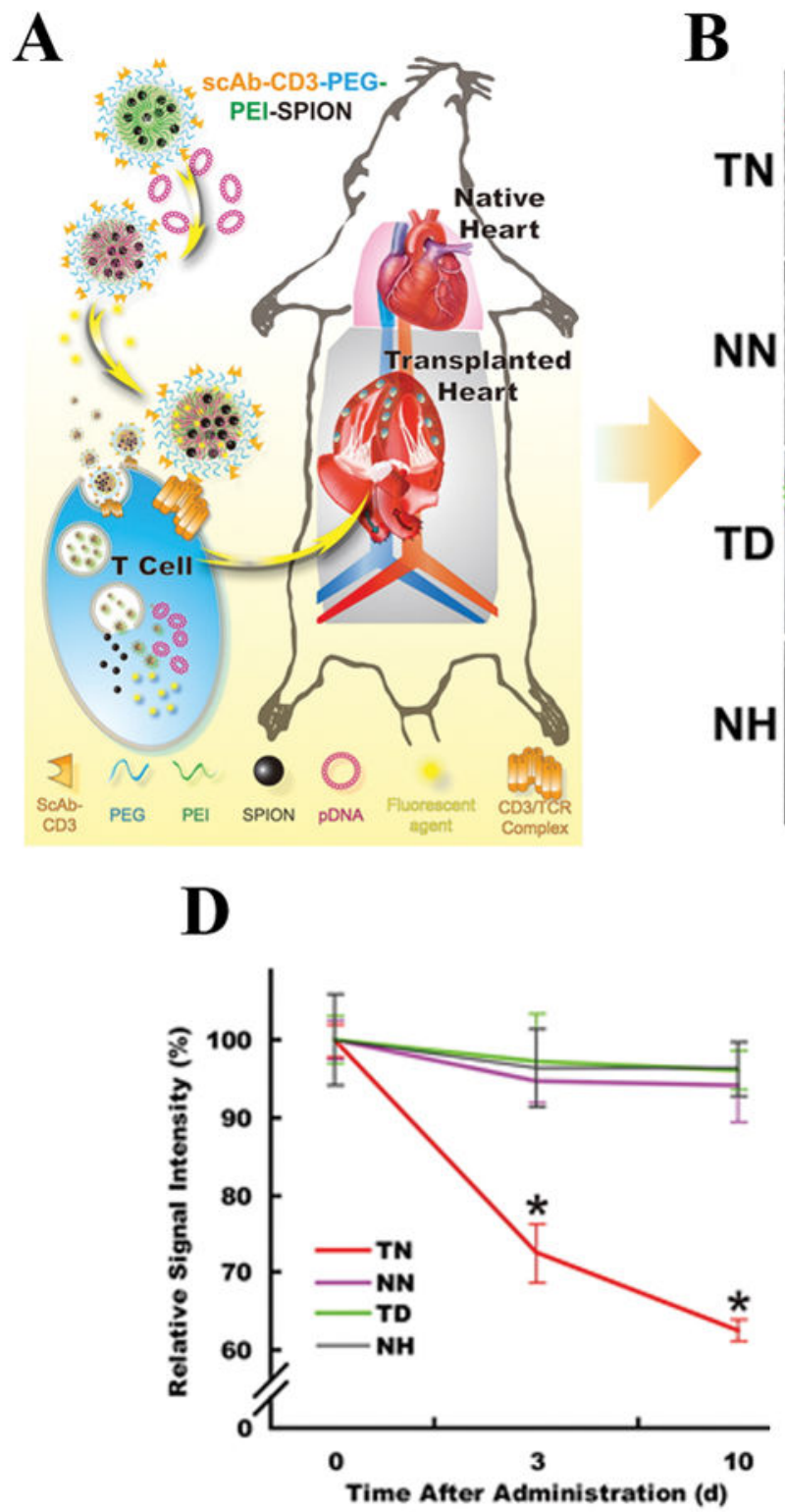
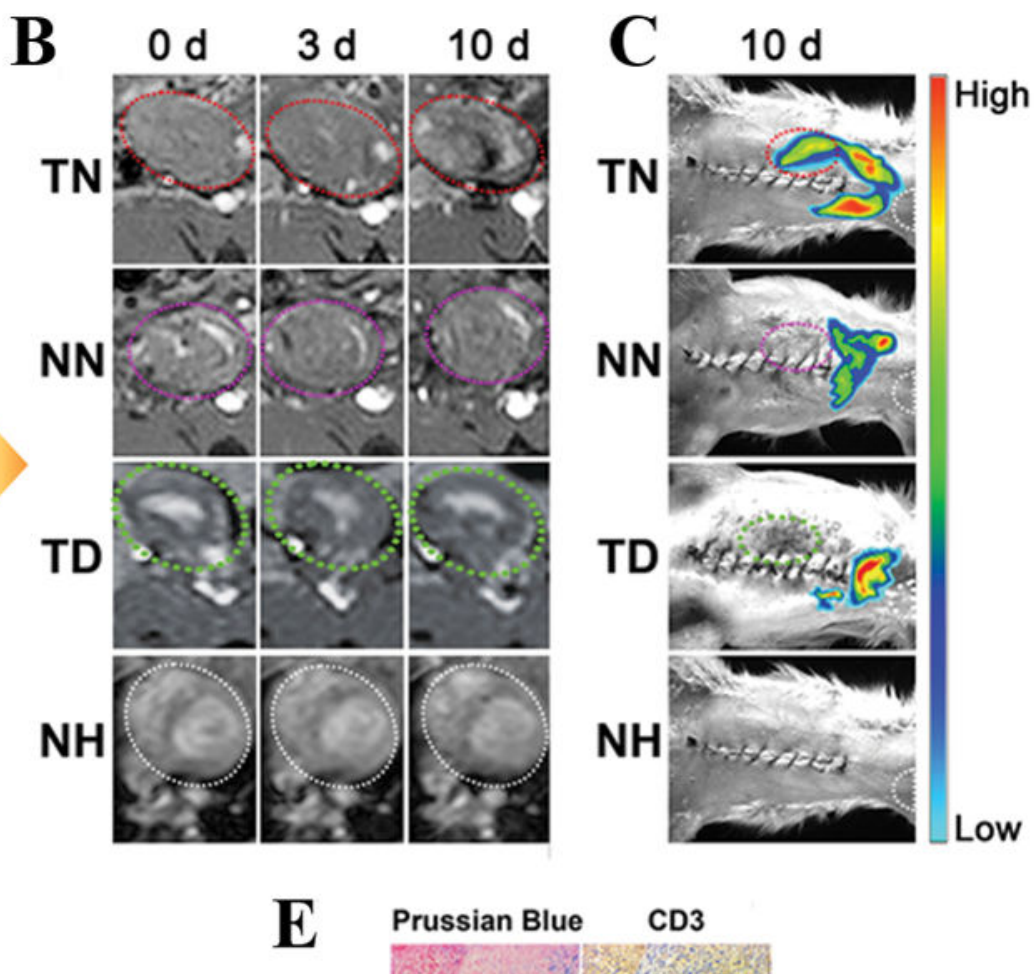

$\mathrm{CD} 3$

TN

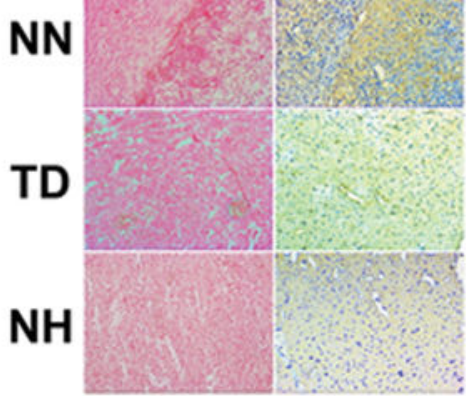

Figure 3.

(A) A schematic diagram of the therapeutic process of magnetic targeting polyplex scAbCD3-PEG-g-PEI-SPION. (B) MRI of a transplantation model during the period from 0 to 10 days after transplantation. Areas marked with dashed red, purple, and green circles illustrate the grafted hearts in the animals with different treatments. TN, scAbCD3-PEG-gPEI-SPION/pDNA treatment; NN, PEG-g-PEI-SPION/pDNA treatment; TD, scAbCD3PEG-g-PEI-SPION/pDNA-DGKR treatment; NH, native hearts. (C) Near-infrared fluorescence (NIRF) imaging of the transplantation model during the tenth day after transplantation. (D) MRI signal intensity change in the transplanted heart during the period from 0 to 10 days after transplantation ( $* \mathrm{P}<0.05$, compared with $\mathrm{NH})$. (E) Prussian blue staining and histological CD3 antibody analysis of the graft tissues from the TN, NN, TD groups, and NH (magnification: 100x) on the 3rd day after transplantation. Positive Prussian 
blue staining is shown in blue, and positive CD3 immunohistochemical staining is shown in brown. Reprinted (adapted) with permission from (Guo, Y.; Chen, W.; Wang, W.; Shen, J.; Guo, R.; Gong, F.; Lin, S.; Cheng, D.; Chen, G.; Shuai, X. Simultaneous diagnosis and gene therapy of immuno-rejection in rat allogeneic heart transplantation model using a $\mathrm{T}$ celltargeted theranostic nanosystem. ACS nano 2012, 6, 10646-10657). Copyright (2012) American Chemical Society. 
(A)

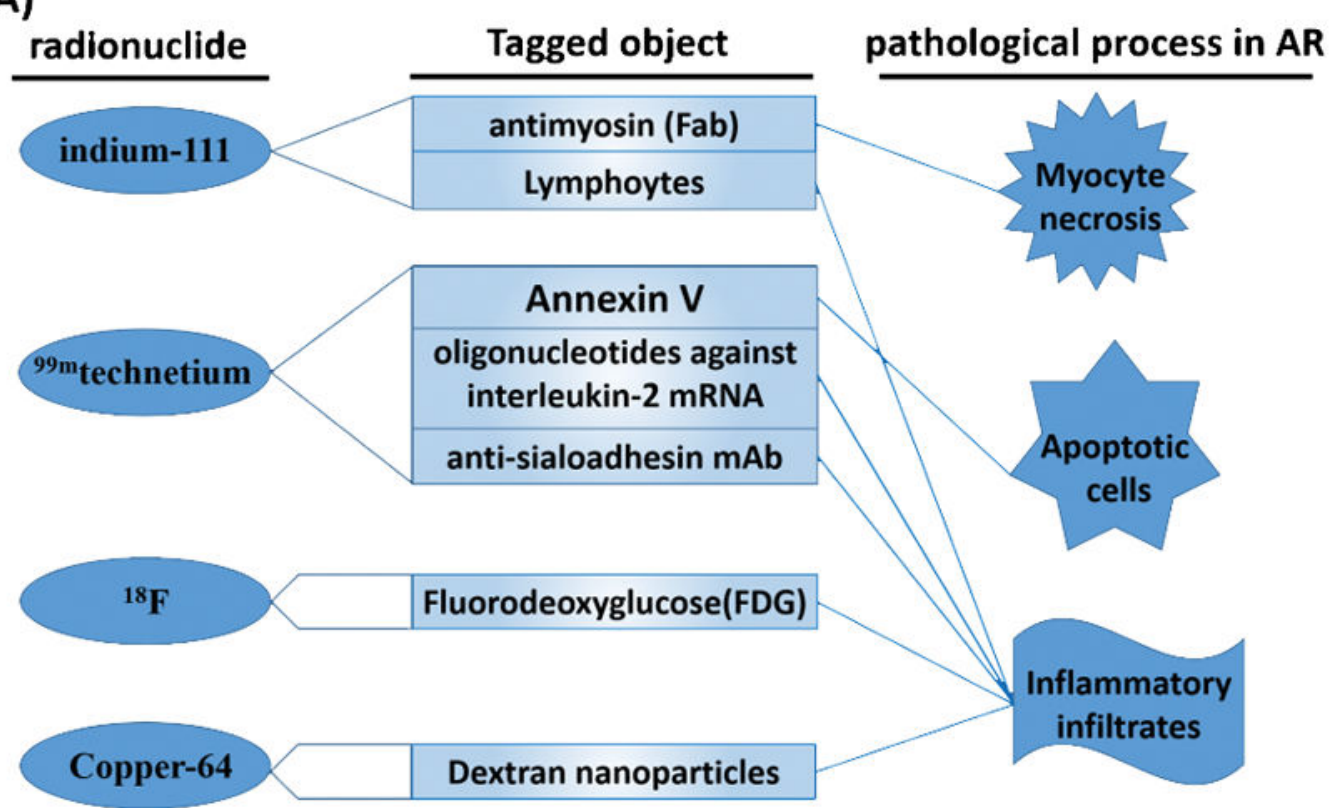

(B)

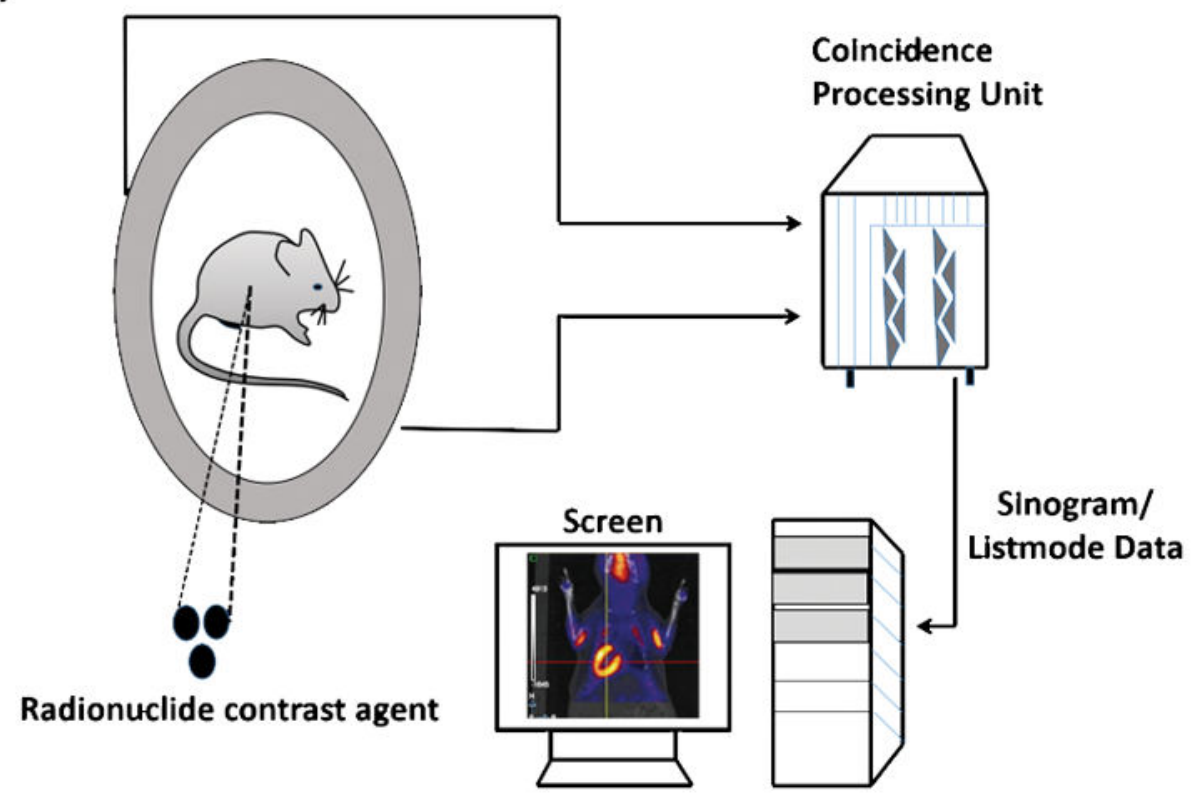

Figure 4.

(A) Radionuclides commonly used in AR detection and their corresponding labelled object that involved in myocyte necrosis, apoptosis and inflammatory infiltrates. (B) The simple flow chart of nuclide imaging in AR. 


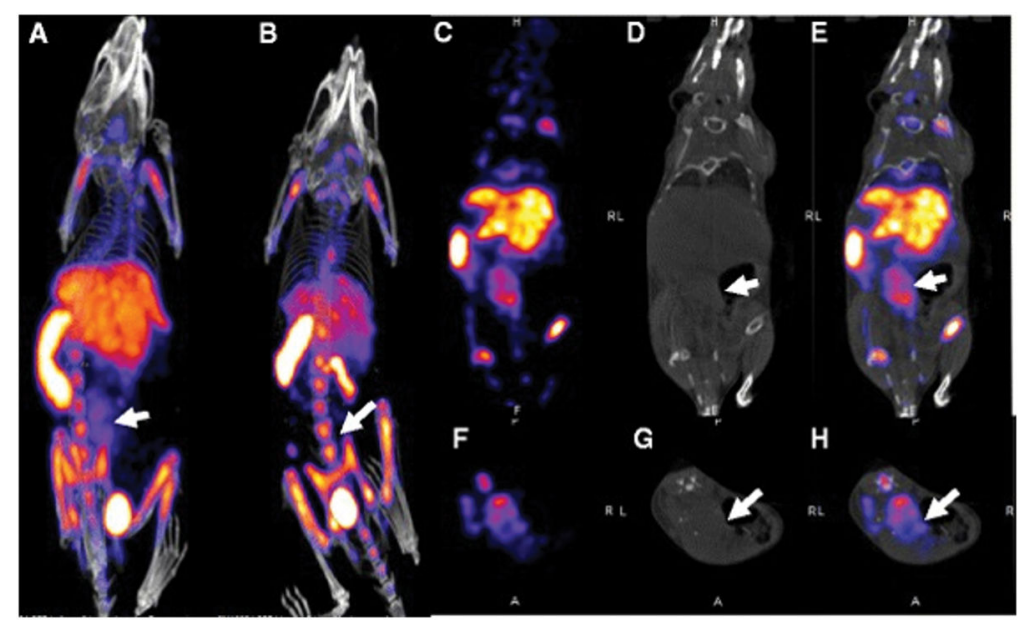

Figure 5.

99mTc pertechnetate-radiolabeled anti-sialoadhesin mAb (SER-4) nanoSPECT/CT imaging of $\mathrm{Sn}+$ macrophages in a heterotopic cardiac transplant model. Representative maximum intensity projection nanoSPECT/CT images of recipients with either a allogeneic or $b$ syngeneic heart grafts that were imaged $3 \mathrm{~h}$ post injection with 99mTc-SER-4. CT, SPECT and fused images of the coronal (c, d, and e, respectively) and transversal (f, $\mathrm{g}$, and $\mathrm{h}$, respectively) planes are also shown. Arrows indicate the site of the abdominal cardiac transplant showing the uptake of tracer in an allogeneic heart graft and its absence in a syngeneic heart graft model. Adapted from O'Neill. ${ }^{49}$ 


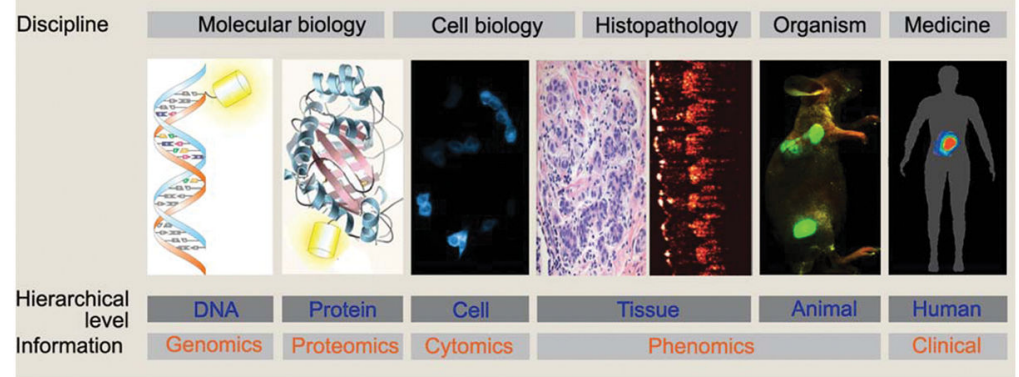

Figure 6.

OMI can be used to investigate the dynamics of biological events in molecules, cells, tissues and organisms in real time and in vivo, with high spatial and temporal resolution. Adapted from Wei Du. ${ }^{56}$ 


\section{Table 1}

Comparison of different imaging modalities in probes and experimental subjects currently used for AR in heart transplantation.

\begin{tabular}{|c|c|c|}
\hline Imaging Modalities & Probes & Experimental Subjects \\
\hline \multirow{2}{*}{ Ultrasound Molecular Imaging } & mcirobubble & rats $^{11,12}$ \\
\hline & nanobubble & rats $^{19}$ \\
\hline \multirow{4}{*}{ Molecular and Cellular MRI } & SPIO & rats $^{37}$ \\
\hline & USPIO & rats $^{22}$ \\
\hline & MPIO & rats $^{23,24}$ \\
\hline & fluorine-19 & rats $^{27}$ \\
\hline \multirow{4}{*}{ Nuclear Imaging } & indium-111 & $\operatorname{dogs}^{43}$, humen $^{44,45}$ \\
\hline & 99m Technetium & mice $^{49}$, rats $^{48}$, humen $^{46,47}$ \\
\hline & {$\left[{ }^{18} \mathrm{~F}\right] \mathrm{FDG}$} & mice $^{52}$ \\
\hline & copper-64 & mice $^{55}$ \\
\hline \multirow{2}{*}{ Optical Molecular Imaging } & luciferase & Mice $^{59,62}$, rats ${ }^{65}$ \\
\hline & fluorescence protease & mice $^{63}$ \\
\hline
\end{tabular}

Transplantation. Author manuscript; available in PMC 2018 September 01. 